\title{
The Setting and its Role in the Evaluation of Gait of Children with Cerebral Palsy
}

\author{
Mariangela Pisasale* and Matteo Cioni
}

\author{
Gait and Movement Analysis Laboratory, Physical Medicine and Rehabilitation, School of Medicine, University of \\ Catania, Catania, Italy
}

\begin{abstract}
Behaviour of children in the gait laboratory can be affected both by the environment and personnel. Therefore, the potential effects of several factors should be considered when a gait laboratory is settled. Therefore, shape, material and color of walls and floor, the position of pictures in the walls, the presence of toys and technological instrumentation can influence children by inducing positive or negative behavioral responses. An environment considered hostile can cause a refuse of walking or a low velocity of walking, which is correlated to low EMG activation patterns.
\end{abstract}

Keywords: Gait, gait laboratory, setting, children, cerebral palsy.

The definition of setting was created by Barker [1] to indicate the environment where an event occurs. The setting can influence the event and consequently psychometric studies of experimental psychology are aware of this phenomenon and try to keep unmodified the setting where a measurement occurs. Regarding the gait analysis, by considering this definition, the environment is represented by the laboratory and the event is the gait evaluation of a child. Then, the aim of this article is to consider the characteristics of the setting of a gait laboratory, the possible influences of setting on gait evaluation and other factors which can influences the interaction between the child and the setting of laboratory.

It is an usual observation for physical therapists, physiatrists and pediatric neurologists that the behavior of children, both with and without cerebral palsy, can be different according to the setting. Then, we can clinically observe a more or less marked abnormal muscle activation, according to psychological conditions of the child that we are examining: if he is confident in us, we can see a lesser dynamic contracture of calf muscles than when he is suspicious about the possible administration of a drug by injection. The obvious consequence is that when we perform a gait evaluation we have to consider some possible influences of setting in what we are recording. Another, consequence of the impact of setting is that children may walk with a different pattern just because they feel to be "under observation" and they want to walk "at the best". Therefore, the walking performance that we record it is not the usual one for that child, but it is "a learned pattern" during the physical therapy sessions. This phenomenon can affect the objectivity of our evaluation and then cause inadequate or wrong therapeutic indications.

*Address correspondence to this author at the Gait and Movement Analysis Laboratory, Physical Medicine and Rehabilitation, School of Medicine, University of Catania, Italy; Tel: +39 95 7384079; Fax: +39 95 7384238; E-mail: marpisa@hotmail.it
When we discuss about the "setting" we should firstly consider the several components of a clinical and/or experimental gait laboratory. In Table $\mathbf{1}$ are reported the intrinsic factors of a laboratory which contribute to compose the environment where a gait evaluation occurs. Firstly, we should consider that laboratories of gait analysis are usually placed within an hospital. Since, most of children have experienced painful experiences in hospitals (usually injections to collect blood samples) we can easily imagine that they do not appreciate to go at hospital, at least until they realize that a gait evaluation does not implicate some painful interventions. However, it should be also considered that sometimes to remove disposable electrodes from skin over some muscles (adductors) can be quite painful for children and then care should be taken to avoid the following negative conditioning: GAIT LAB = PAIN. A second component of laboratory setting is represented by people working on it. This could be considered a positive one, because they have the opportunity to well interact with child and parents and, by explaining what it will be done, they can restore confidence of them. Possibly, both physiatrists and physical therapists should avoid to worn a white lab coat, which could remember painful intramuscular injections.

Table 1. Factors Composing the Setting of a Gait Laboratory

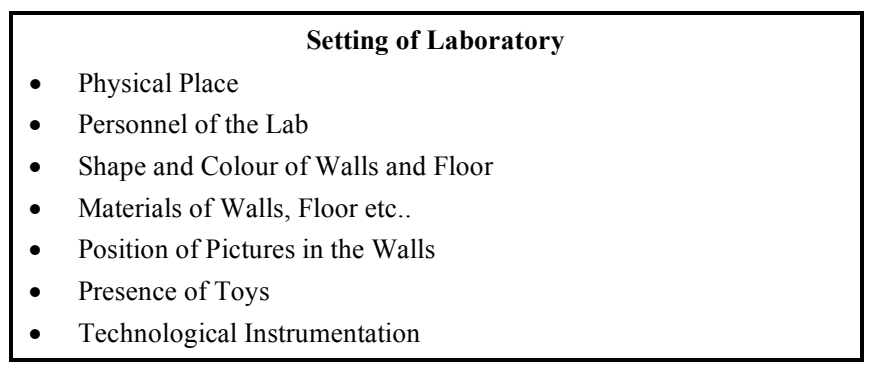

The shape, size and color of the laboratory are responsible for the first emotive imprinting of the child. In fact, we should always remember that children are shorter in size than adults (about $1 / 4$ or $1 / 2$ an adult) and then, in a large place as a gait laboratory, they can feel to be without a 
spatial reference point. For this reason, shape and colors should be chosen to give the impression that the room where the lab is located is smaller than the real size. Furthermore, cartoons and other pictures should be placed at about $100 \mathrm{~cm}$ from the floor to give them some known information. The interior decorations and furnishings of the laboratory are quite different from those of apartments where the child leaves. In fact, metals and plastics are materials utilized in the laboratories instead of woods at home. Consequently, interiors of laboratory can be considered scarcely familiar in respect to those of school or home and the laboratory can be seen quite "cold".

The best way to introduce positively the child to laboratory is to give him the possibility to play with traditional toys or electronic play games. By this recreational approach even the most opposing child can be convinced to participate to the gait evaluation. In fact, we should always remember that a gait evaluation regards an active voluntary performance and if we do not have the child's collaboration we cannot perform any evaluation and it is better to try again in another day. Another positive factor to obtain the collaboration of children is the presence of high technological level instruments. In fact, nowadays children live in houses equipped with highly sophisticated household appliances or other electronic instrumentation as LCD or LED televisions, video recorders, monitors, cameras, play games, mobile phones and personal computers. Then, they find very interesting to spend some time in a gait laboratory and then they could be prompted to go back again in this technological place.

The impact of the child with the setting of the laboratory can be influenced by other factors as advices/instructions given from the parents about the quality of walking. In fact, most of them invite them to "walk well" and to get a good impression. Then, both the new environment and the parent's words contribute to stiff the walking of these children. Consequently in some cases it is possible to record a walking pattern which is completely different from the usual one. Another consideration should be done about the influence of the setting on the child. In our clinical and laboratory practice we have observed that children with cerebral palsy have a better interaction with the laboratory than others typically developing. In fact, the former are more willing to participate and to collaborate with the examiner, whilst the latter are often naughty and scarcely collaborative. In our mind, this phenomenon could mean that children with cerebral palsy are used to have relationships with rehabilitation's settings and that also they are at the top of attention in these settings. By contrast, those infant typically developing are scarcely trained to be the object of specific treatments or evaluations.

\section{REFERENCE}

[1] Barker RG. Ecological Psychology: Concepts and methods for studying the environment of human behavior. California, USA: Stanford University Press 1968. 